\title{
Thresholds, Bifurcations and Mental Control: An Application of Nonlinear Dynamics to Psychotherapy
}

\author{
Keith Warren, Ph.D. \\ University of Wisconsin-Madison School of Social Work \\ Julien C. Sprott, Ph.D. \\ University of Wisconsin-Madison Physics Department
}

\subsection{Introduction}

Mental control paradoxes-those times when we try to control a thought or behavior and the effort at control becomes counterproductive-are both common and enigmatic. Why is it so difficult to stand in a corner and refrain from thinking of a white bear-but only if we have been asked to do so [Wegner, 1989]? Why, when we try to control our thoughts and behaviors, do they so often spin out of control?

These are questions of more than theoretical significance. A variety of mental illnesses manifest themselves in the form of thoughts and/or behaviors that defy control. Examples include eating disorders, some sex offending behavior, obsessive compulsive disorder, and pathological gambling [Hollander, 1993]. A better understanding of mental control paradoxes might yield valuable insights into them all. Further, mental control paradoxes might be involved in the more general phenomenon of clinical resistance, in which psychotherapy clients with a variety of problems experience difficulty in changing their thoughts or behaviors [Kirsch \& Lynn, 1999].

In this paper, we will review and critique some of the contemporary literature on mental control paradoxes. We will then develop a simple nonlinear model that produces mental control paradoxes as the result of a bifurcation, and which answers the criticisms that we propose. We will demonstrate an application of the model to the self-rated intensity of ruminations in an adult psychotherapy client. Finally, we will discuss the implications of this model of mental control paradoxes.

\subsection{Current Models of Mental and Behavioral Control Paradoxes}

\subsubsection{A brief overview of current models}

The last decade has brought an upswing in the study of mental control paradoxes, and several theories as to how they might arise. For instance, Martin \& Tesser [1996] have argued that ruminative thoughts, which often do not respond to efforts at control, arise from goal frustration. In order to attain a goal, people must think about the goal and ways of attaining it. If something frustrates the attainment of the goal, there is a tendency for thoughts pertaining to the goal to continue. There is empirical support for this position dating back to the 1930s, drawn from experiments in which the ability of 


\section{Thresholds, Bifurcations and Mental Control}

subjects to reach goals is systematically frustrated. In many, although not all, of these experiments, the subjects in the goal frustration condition tended to ruminate about the goal. Martin and Tesser argue that the mixed results arise from other variables that affect rumination, such as subjects' theories that attempt to explain goal frustration.

Others have drawn on cybernetic theory to explain mental control paradoxes. Wegner and Wenzlaff [1996; see also Wegner, 1994] write that the mental control process requires both an operating process that seeks to create the desired state, and a monitoring process that tells whether the desired state has been obtained.

However, the monitoring and control processes tend to operate at cross-purposes. While the mental control process is conscious, the monitoring process occurs outside of conscious control. The mental control process searches for and seeks to implement the desired mental state, while the monitoring process searches for the undesired mental state, so as to be sure that it is really gone. The upshot is that the unconscious monitoring process tends to bring the undesired mental state back to awareness [Wegner, 1994; Wegner \& Wenzlaff, 1996]. The effect should be more powerful when cognitive load weakens the conscious, mental control process [Wegner, 1994; Wegner, Broome \& Blumberg, 1997], and experiments have documented this effect [Wegner, Broome \& Blumberg, 1997].

\subsubsection{Difficulties with current models of mental control paradoxes}

While current theories demonstrate why it might be difficult to suppress an unwanted thought or behavior, they do not explain why an attempt at suppression or control might lead to an actual increase in the intensity of the unwanted thoughts or behaviors. Why does the ironic monitoring process, for instance, not simply bring the unwanted thought or behavior back at a low level? Why would an attempt at suppressing a thought produce an increase in the level of the thought, rather than simply failing and giving results indistinguishable from those subjects who did not attempt to suppress the thought?

An actual increase in unwanted thoughts is a common finding in studies of mental control paradoxes. For instance, Wegner and Gold [1995] asked subjects to suppress thoughts of "old flames"-people with whom the subjects had had romantic involvement that had ended by the time of the experiment. Subjects were asked to report their thoughts, and their skin conductance level (SCL) was monitored as an indicator of emotional reactivity. Subjects who attempted to repress thoughts of old flames subsequently experienced either an increase in SCL or an increase in the number of thoughts of the old flame, depending on how long ago the relationship had ended. An earlier study [Wegner, Shortt, Blake \& Page, 1990] had found increases in SCL in subjects who tried to suppress thoughts of sex. And Wegner, Schneider, Carter $\&$ White [1987] found an increase in the thought of a white bear following attempts to suppress the thought.

It therefore seems that, while contemporary theories of mental control paradoxes mark an important advance in understanding, they do not completely explain the phenomenon. They offer explanations as to why it might be difficult to completely rid oneself of an unwanted thought or behavior, but they fail to explain why the effort can actually lead to an increase.

A further difficulty with contemporary models of mental control paradoxes is pragmatic in nature. It is difficult to see how a theory that assumes an unconscious, and therefore unobservable, process can ever be observed in a naturalistic setting. This has meant that the empirical investigation of mental control paradoxes has been confined to laboratory experiments in which researchers can, by experimental manipulations, adduce indirect evidence of the occurrence of a paradox. This is unfortunate, because one reason for the current interest in mental control paradoxes is their potential importance in explaining clinical phenomena such as client resistance to change, and evidence of mental control paradoxes in clinical settings would be an important step toward such an explanation [Kirsch \& Lynn, 1999]. 


\section{Thresholds, Bifurcations and Mental Control}

We believe that a nonlinear model of mental control paradoxes would address both the theoretical and pragmatic difficulties inherent in current models of this phenomenon. We think that the increase in a problem behavior that is the hallmark of a mental control paradox can be reasonably modeled as a bifurcation in a nonlinear system. Further, an explicit mathematical model would be amenable to statistical analysis using clinical data, a useful feature that would allow the study of mental control paradoxes in naturalistic settings.

\subsection{A Nonlinear Model of Mental and Behavioral Control Paradoxes}

In this section, we would like to propose a mechanism that would lead to nonlinearity in the way in which thoughts or behaviors fluctuate over time, and then develop a simple mathematical model that will allow us to demonstrate how a mental control paradox could arise out of such nonlinearity.

We will begin by assuming that an individual suffers from thoughts or behaviors that he or she might want to control. One possibility might be obsessive ruminations, such as thoughts of intense anger toward others. Once the individual begins rumination he/she is likely to continue [Martin \& Tesser, 1996]. Thus, the rumination forms a dynamical system; what happens in one time period affects what happens in the next [Devaney, 1992].

There is evidence that people find such ruminations to be unpleasant [Steketee, 1993]. Moreover, angry ruminations can lead to outbursts and confrontations that can disrupt friendships or working relationships. Thus, the individual will be motivated to control his/her ruminations, perhaps by engaging in a distracting behavior such as listening to music or simply by trying to substitute other, happier thoughts [Wegner, 1989]. However, the individual is likely to be more highly motivated to control the ruminations when they are causing considerable discomfort, or when they have led to an outburst that has disrupted his/her relationships. Another way of putting this is that the individual is likely to be more highly motivated to control his/her ruminations when they are at a higher level of intensity.

A piecewise linear difference equation, known in statistics as a threshold autoregression model or TAR [Hansen, 1997; Tong, 1990] offers a simple way to model such a situation. Such an equation models a time series using two linear regimes separated by a threshold [Tong, 1990]:

$X_{t}=a+b X_{t-1}$ if $X_{t-1}$ falls below the threshold value

$X_{t}=c+d X_{t-1}$ if $X_{t-1}$ falls above the threshold value [eq. 1]

In order to apply this model, it is necessary to assume that human thoughts or behaviors can be numbered on some consistent scale; numerous scales for this purpose do in fact exist [Rubin \& Babbie, 1997]. X denotes the level of the problematic thought or behavior - in this case, angry ruminations. The subscript, $t$ or $t-1$, indicates the time period. In the situation that we are modeling, if the problem thought or behavior rises above the threshold in one time period, the individual uses a control strategy in an attempt to lower it in the next.

Such a model will include two equilibrium points, one for the equation below the threshold and one for the equation above the threshold. Such equilibrium points can be stable or unstable, depending on the absolute value of slope of the function; if that value is greater than one, the slope is unstable, while if it is less than one the slope is stable [Devaney, 1992]. If we assume a slow rise in anger, followed by a successful attempt to lower the anger when the level passes the threshold, the equation might look like this:

$\mathrm{X}_{\mathrm{t}}=.4+.2 \mathrm{X}_{\mathrm{t}-1}$ if $\mathrm{X}_{\mathrm{t}-1}$ falls below the threshold value

$\mathrm{X}_{\mathrm{t}}=3-1.5 \mathrm{X}_{\mathrm{t}-1}$ if $\mathrm{X}_{\mathrm{t}-1}$ falls above the threshold value [eq. 2] 


\section{Thresholds, Bifurcations and Mental Control}

It is straightforward to solve for the equilibrium values above and below the threshold. The equilibrium value below the threshold is equal to .5 , while the equilibrium value above the threshold is equal to 1.2. The equilibrium point below the threshold is stable, while that above the threshold is unstable. We will assume for the sake of this example that the threshold falls at an "anger level" of 1 . In this case, when the model is iterated the level of anger will tend to converge on the stable equilibrium point at $\mathrm{X}_{\mathrm{t}}=\mathrm{X}_{\mathrm{t}-1}=.5$.

Now, let us suppose that this particular individual decides that this level of anger is unacceptable, and therefore decides to employ the control strategy at an anger level of .2. We can model this by lowering the threshold to .2. The new threshold is lower than the sole stable equilibrium point for the system as a whole; thus, the system can no longer reach a stable equilibrium point.

Since the one remaining equilibrium point is unstable, the destruction of the stable equilibrium point brings about a bifurcation into either a periodic or chaotic state. In this case the level of anger will repeatedly jump above the threshold, reaching values higher than the original equilibrium. Thus, an attempt to control anger has actually led to a series of jumps in the level of anger. A mental control paradox has occurred.

It should be noted that other model configurations leading to mental control paradoxes are possible. If a first difference is taken to induce stationarity in the time series, the foregoing discussion remains valid, but a mental control paradox will lead to jumps in the level of increase of the problem behavior. If the slope parameter above the threshold yields a stable equilibrium point, lowering the threshold will produce a jump to the higher equilibrium point. On the other hand, it is possible to have a stable slope parameter with a negative intercept value. (Negative values are common in the case of a time series of changes from one period to the next, as occurs after taking the first difference of a time series.) Such a configuration can yield a stable equilibrium point for values above the threshold that is actually located in the regime below the threshold, and possibly below the equilibrium point for values below the threshold. This can also bring about a bifurcation into a periodic regime if the threshold falls between the two equilibrium points.

It has been noted that mental control paradoxes appear more likely under conditions of stress [Wegner, 1994; Wegner, Broome \& Brumberg, 1996]. In the laboratory, psychologists typically manipulate the stress level by assigning subjects to do a second task along with the main task, thus increasing cognitive load, or by varying their descriptions of the importance of the task, thus increasing subjective stress level. One could simulate this in the nonlinear model of mental control paradoxes by adding a noise component to the model. We would expect the addition of white noise to increase the number of jumps across the threshold, even if the threshold does not fall below the equilibrium point in the lower regime. This would be an example of the well-known instability of nonlinear systems in noisy conditions [Arecchi, 1987].

\subsubsection{Empirical application of the model}

One of the reasons for developing this model was to begin to study mental control paradoxes outside of the laboratory. In this section we will briefly describe an application of the model to the self-recorded level of anxiety of a psychotherapy client, with the hope of demonstrating the plausibility of the model in an empirical setting. The participant was an adult male. The participant was in therapy for anxiety at the time, and carried a DSM Axis I diagnosis of generalized anxiety disorder. For 274 days the participant drew a line that tracked the intensity of his anxiety, beginning in the morning when he woke and ending when he went to bed at night, on a self-anchored scale ranging from one to ten. The data were collected for purposes of monitoring 


\section{Thresholds, Bifurcations and Mental Control}

changes in the level of his anxiety. ${ }^{1}$ For this analysis, we recorded the participant's daily high rating of the intensity of his anxiety. The daily high was chosen because it seemed likely that the subject would condition his actions on his worst, and therefore most memorable, time of the previous day. The time series of daily highs in level of anxiety is shown in Figure 1.

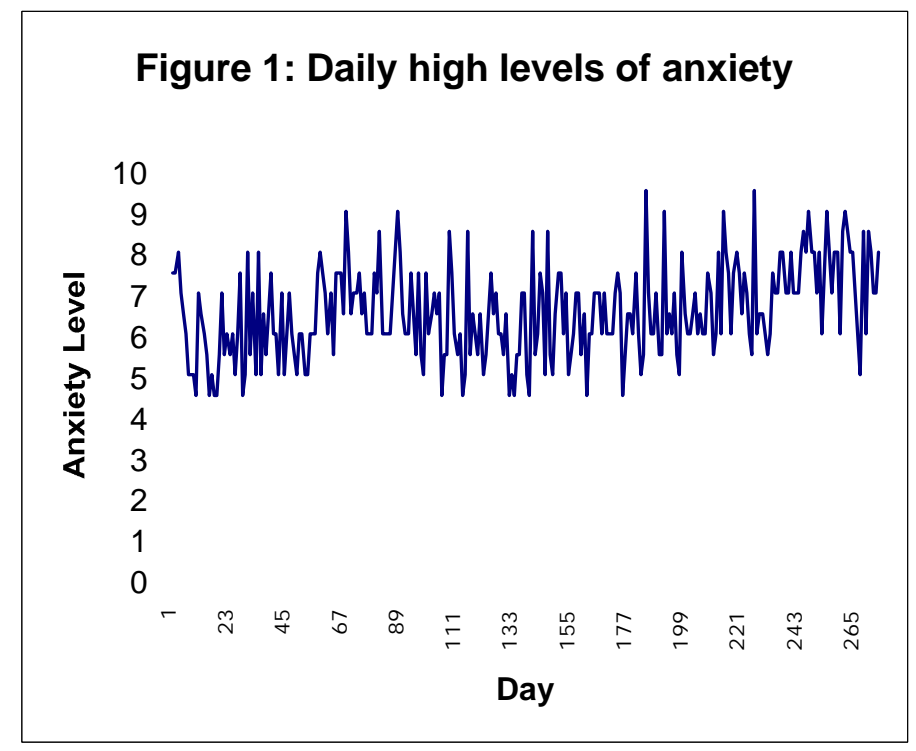

A first difference was applied to this data set to induce stationarity. The firstdifferenced time series was analyzed using the bootstrap methodology suggested in Hansen, 1997. In this analysis linearity is the null hypothesis, with the threshold model tested as the alternative hypothesis. Because the linear model is equivalent to a threshold model with zero thresholds, this is equivalent to testing nested models (Hansen, 1999). The results are reported in Table 1.

Table 1: Evidence of nonlinearity in daily self-reported intensity of anxiety (Estimated on $1^{\text {st }}$ difference of series)

Threshold Estimate: 0

95\% Confidence Interval: $-2.5,3.0$

Regime $1, \mathrm{X}_{\mathrm{t}-1} \leq 0$ :

\begin{tabular}{|l|l|l|}
\hline Parameter & $\begin{array}{l}\text { Parameter } \\
\text { Estimate }\end{array}$ & Standard Error \\
\hline Constant (a) & .15 & .14 \\
\hline $\mathrm{X}(\mathrm{t}-1) \quad(\mathrm{b})$ & -.25 & .10 \\
\hline
\end{tabular}

Regime 2, $\mathrm{X}_{\mathrm{t}-1}>0$ :

\begin{tabular}{|l|l|l|}
\hline Parameter & $\begin{array}{l}\text { Parameter } \\
\text { Estimate }\end{array}$ & Standard Error \\
\hline Constant (c) & .53 & .20 \\
\hline $\mathrm{X}(\mathrm{t}-1) \quad(\mathrm{d})$ & -.82 & .10 \\
\hline
\end{tabular}

Joint $\mathrm{R}^{2}=.24$

F-test for no threshold: 12.04

Bootstrap P value: .01

The results in the table are consistent with a situation in which mental control paradoxes could arise due to nonlinearity in human behaviors. A threshold appears to

\footnotetext{
${ }^{1}$ A more complete account of this study can be found in Warren, Sprott \& Hawkins (in preparation).
} 


\section{Thresholds, Bifurcations and Mental Control}

exist. The regime below the threshold includes a stable equilibrium point. The regime above the threshold includes an equilibrium point that might be stable or unstable- the confidence interval around the slope parameter is too wide to give a clear indication. In this case, the estimated threshold value of zero is slightly below the estimated equilibrium point for values below the threshold $(X \cong .12)$ and so a sudden jump leading to increased levels of anxiety appears plausible. (It is, of course, possible for dynamical systems with multiple stable equilibria to become unstable when affected by environmental noise (Arecchi, 1987).) A bifurcation leading to increased volatility seems less likely, since the equilibrium point in the regime above the threshold is stable-i.e., less than one in absolute value-for most of the values within two standard errors of the estimated parameter. Numerical simulations with a slightly unstable parameter value above the threshold $\left(X_{t-1}=1.02\right)$ yielded a pattern of oscillations that showed a slow increase in amplitude followed by a sudden drop, a cycle that repeated itself over a period of about one hundred days. The oscillations were in some cases weakly chaotic, with a Lyapunov exponent of about .05 .

Needless to say, evidence of nonlinearity in one time series is far from proof that nonlinearity in human behavior is common, and even further from proof that nonlinearity leads to mental control paradoxes. At present, there is evidence of nonlinearity in the behaviors of adolescent sex offenders (Warren \& Knox, forthcoming), daily fluctuations in the level of self-recorded intensity of obsessive thoughts (Warren, Sprott and Hawkins, in preparation) and daily fluctuations in the amount of alcohol consumed by an individual with a diagnosis of substance abuse (Warren, Hawkins and Sprott, in preparation). All of these are, however, exploratory studies, carried out on between one and three time series. It is thus difficult to say how common nonlinearity in problem thoughts or behaviors might be. Work with larger data sets would help in this regard. A larger data set might also allow a statistical test as to whether nonlinearity can lead to a mental control paradox since, according to this theory, the volatility and number of problem thoughts or behaviors should increase as the threshold value decreases.

\subsection{Discussion and implications}

While the empirical study of nonlinearity and mental control paradoxes is still in an exploratory stage, we believe that this line of research carries potentially significant implications for applied social science researchers and practitioners. For instance, research on the effectiveness of clinical interventions is typically carried out using experimental designs and evaluated using linear statistics such as analysis of variance [Rubin and Babbie, 1997]. Nonlinear time series structure, which may show no structure at all when analyzed with linear statistics [LeBaron, 1999] represents a likely source of random error in such studies. This is particularly problematic, because some clinically relevant behaviors might be more highly nonlinear, and therefore more noisy, than others. The time series structure of problem behaviors, however, remains largely unexplored.

In general, evidence of nonlinearity in human behaviors implies an increased focus on the variance of those behaviors, since a change in the parameters of a nonlinear system can lead to a change in the variance of its output [Thelen \& Smith, 1994]. Again, the effect of interventions on the variance of behaviors remains largely unexplored; clinical studies typically focus on changes in the mean [Rubin and Babbie, 1997]. Many clinical researchers would probably be puzzled as to why one should care about variance at all. One answer, in line with the argument of the preceding paragraph, is that it will be more difficult to demonstrate the effectiveness on an intervention that increases the variance of a behavior. Another answer is that variance over time is an index of the stability of a given behavior, and that high levels of instability should make it more difficult to plan and execute an intervention. 


\section{Thresholds, Bifurcations and Mental Control}

A nonlinear theory of mental control paradoxes also focuses attention on the dynamics of treatment, with the implication that the timing of an intervention may be as important as the content of the intervention in facilitating change. In fact, a poorly timed intervention, in this case one that aims to control a problematic thought, emotion or behavior after an initial rise, runs a risk of adding to the instability and increasing the severity of the problem. A good example of an intervention that follows a rise in problem behavior is thought stopping, in which individuals who suffer from obsessive ruminations are taught to think, "Stop!" as a means of controlling the ruminations. Evidence of the effectiveness of thought stopping has been decidedly mixed. In some cases it appears to be effective, while in others it appears to make problems worse [Wegner, 1989]. In the current model, if thought stopping has an effect, it will create a threshold nonlinearity. Above the threshold, thought stopping will indeed tend to lower the intensity of ruminations. But its very success can work against it; it will become tempting to lower the threshold, trying to stop the ruminative thoughts sooner, which can lead to a bifurcation and increased instability. The nonlinear theory of mental control paradoxes implies that thought stopping, if used at all, might work best as an intervention that would only follow particularly sharp increases in the problem thought.

The interventions that contrast most sharply with thought stopping are paradoxical interventions, in which the therapist asks a client to produce more of the problem thought or behavior [Wegner, 1989]. ${ }^{2}$ In the context of the current model, these interventions encourage clients to raise the threshold of the control regime, moving away from the possibility of a mental control paradox and tending to stabilize the problem behavior at a low level. There is evidence that paradoxical interventions can be effective in treating insomnia [Ladocouer \& Gros-Louis, 1986] and anxiety [Ascher and Schotte, 1999] among other problems. However, their effectiveness appears to depend on the dynamics of the problem. For instance, there is evidence that paradoxical treatment of anxiety works best when the anxiety is "recursive," i.e., when individuals are anxious about getting anxious [Ascher and Schotte, 1999]. A nonlinear model of mental control paradoxes might be helpful in sorting out situations in which paradoxical interventions would and would not be expected to be effective.

The nonlinear model that we have described does not so much challenge contemporary models of mental control paradoxes as supplement them by changing the focus of inquiry and understanding. The ironic process model, for instance, is reductionist in nature; it posits an underlying control process that results in mental control paradoxes [Wegner, 1994]. While we believe that the nonlinear model has certain advantages (it produces an increase in the problem thought, emotion or behavior and is more mathematically explicit and therefore in principle testable using clinical data) it fails to posit an underlying process. In this sense the two models can be seen as complementary. This, in turn, alters the social scientific question we ask when comparing the two models. Rather than asking which of the two models is correct, it might be more reasonable to ask which of the two models is the more useful when trying to understand a given phenomenon. When we are trying to understand sudden changes in human behavior, nonlinear models will prove to be useful and, perhaps, necessary.

\section{References}

Arecchi, F.T., 1987, Hyperchaos and 1/F Spectra in Nonlinear Dynamics, in Chaos, Noise and Fractals, edited by E.R. Pike and L.A. Lugiato, Malvern Physics Series, Adam Hilger (New York).

\footnotetext{
${ }^{2}$ This is a very generic, and considerably oversimplified, account of paradoxical interventions, which in fact show considerable variety.
} 


\section{Thresholds, Bifurcations and Mental Control}

Ascher, L.M. \& Schotte, D.E., 1999, Paradoxical Intention and Recursive Anxiety, Journal of Behavior Therapy and Experimental Psychiatry, 30(2), 71-79.

Devaney, R. L., 1992, A first course in chaotic dynamical systems, Addison-Wesley (Reading, MA).

Hansen, B. E., 1997, Inference in TAR Models. Studies in nonlinear dynamics and econometrics, 2(1) 1-14.

Hansen, B. E., 1999, Testing for linearity. Social Systems Research Institute Working Paper \#9907.

Hollander, E. (ed.), 1993, Obsessive-Compulsive Related Disorders, American Psychiatric Press (Washington, D. C).

Kirsch, I. \& Lynn, S.J., 1999, Automaticity in Clinical Psychology, American Psychologist, 54(7), 504-515.

Ladocouer, R. \& Gros-Louis, Y., 1986, Paradoxical Invention vs Stimulus Control in the Treatment of Severe Insomnia, Journal of Behavior Therapy and Experimental Psychiatry, 17(4): 267-269.

LeBaron, B., 1999, Time series analysis, Seminar held on October 6, 1999 as part of the New England Complex Systems Institute Fall intensive course in complex systems.

Martin, L.L. \& Tesser, A., 1996, Some Ruminative Thoughts, in Ruminative Thoughts, edited by R.S. Wyer, Jr., Advances in Social Cognition, Volume IX, Lawrence Erlbaum (Mahwah, NJ).

Rubin, A. \& Babbie, E., 1997, Research Methods for Social Work (3rd ed.), Brooks/Cole (Pacific Grove, CA).

Steketee, G.S., 1993, Treatment of Obsessive Compulsive Disorder, Treatment Manuals for Practitioners, Guilford (New York).

Thelen, E. \& Smith, L., 1994, A Dynamic Systems Approach to the Development of Cognition and Action, MIT Press (Cambridge, MA).

Tong, H., 1990, Non-linear Time Series Analysis: A Dynamical System Approach, Oxford Statistical Science Series, Oxford University Press (New York).

Warren, K., Hawkins, R. \& Sprott, J.C., in preparation, Evidence of Nonlinearity in the Drinking Behaviors of an Adult Substance Abuser.

Warren, K. \& Knox, K., forthcoming, Offense Cycles, Thresholds, and Bifurcations: Applying Dynamical Systems Theory to the Behaviors of Adolescent Sex Offenders, Journal of Social Service Research.

Warren, K., Sprott, J.C. \& Hawkins, R., in preparation, Controlling the Uncontrollable: Nonlinearity, Bifurcations and Paradoxes in Mental Control.

Wegner, D.M., 1989, White Bears and Other Unwanted Thoughts: Suppression, Obsession, and the Psychology of Mental Control, Viking (New York).

Wegner, D.M., 1994, Ironic Processes of Mental Control, Psychological Review, 101(1), 34-52.

Wegner, D.M., Broome, A. \& Blumberg, S.J., 1997, Ironic Effects of Trying to Relax Under Stress, Behaviour Research and Therapy, 35(1), 11-21.

Wegner, D.M. \& Gold, D.B., 1995, Fanning Old Flames: Emotional and Cognitive Effects of Suppressing Thoughts of a Past Relationship, Journal of Personality and Social Psychology, 68(5), 782-792.

Wegner, D.M., Schneider, D.J., Carter, S.R., White, T.L., 1987, Paradoxical Effects of Thought Suppression, Journal of Personality and Social Psychology, 53(1), 5-13.

Wegner, D.M., Shortt, J.W., Blake, A.W., \& Page, M.S., 1990, The Suppression of Exciting Thoughts, Journal of Personality and Social Psychology, 58(3), 409-418.

Wegner, D.M. \& Wenzlaff, R.M., 1996, Mental Control, in Social Psychology: Handbook of Basic Principles, edited by E.T. Higgens \& A.W. Kruglanski, Guilford Press (New York). 